\title{
Doenças sensíveis ao clima no Brasil e no mundo: revisão sistemática
}

\author{
Tatiane Cristina Moraes de Sousa, ${ }^{1}$ Flavia Amancio, ${ }^{1}$ Sandra de Sousa Hacon ${ }^{1}$ \\ e Christovam Barcellos ${ }^{2}$
}

Como citar

Sousa TCM, Amancio F, Hacon SS, Barcellos C. Doenças sensíveis ao clima no Brasil e no mundo: revisão sistemática. Rev Panam Salud Publica. 2018;42:e85. https:/ / doi.org/10.26633/RPSP.2018.85

RESUMO Objetivos. Fazer um levantamento da literatura existente acerca das doenças sensíveis ao clima (DSC) e dos impactos das alterações climáticas sobre a saúde.

Método. A revisão sistemática foi conduzida conforme a metodologia PRISMA. As buscas foram realizadas nas bases LILACS, PubMed, Scopus e SciELO em julho de 2017, sem restrição temporal. Em todas as bases utilizou-se a seguinte estratégia de busca: (climate) AND (disease) AND (sensitive). As buscas foram realizadas em inglês, espanhol e português.

Resultados. Foram selecionadas 106 publicações. As doenças mais estudadas foram dengue, malária e doenças respiratórias e cardiovasculares. As variáveis climáticas mais estudadas foram temperatura e precipitação. Os estudos mostraram uma relação entre a incidência de determinadas de doenças, principalmente doenças cardiovasculares e respiratórias, dengue, malária e arboviroses, e as condições climáticas em diferentes regiões do mundo. Essa relação foi analisada considerando tanto dados pretéritos de incidência de doenças e variáveis climáticas como pela projeção futura de incidência de doenças de acordo com variações previstas do clima. Identificou-se um número maior de estudos realizados por autores oriundos de países desenvolvidos. Os locais estudados com maior frequência foram China, Estados Unidos, Austrália e Brasil.

Conclusões. Apesar do aumento no número de artigos publicados sobre o tema é preciso enfocar um número maior de variáveis climáticas e ambientais e expandir os estudos para outras regiões do globo.

Palavras-chave Mudanças climáticas; efeitos do clima; dengue; malária; doenças respiratórias; doenças cardiovasculares; Brasil

As evidências de alterações climáticas em virtude da emissão atmosférica de gases de efeito estufa (GEE) têm despertado a atenção acerca do impacto dessas mudanças climáticas sobre diferentes fatores, entre eles a saúde humana (1).
Desde a revolução industrial, a temperatura média da Terra aumentou cerca de $1^{\circ} \mathrm{C}$, gerando fenômenos como desertificação, alterações no ciclo hidrológico e aumento da frequência e da intensidade de eventos climáticos extremos (2).
Segundo o Quinto Relatório do Painel Intergovernamental das Mudanças Climáticas (IPCC) (1), é provável um aumento de 0,3 a $0,7^{\circ} \mathrm{C}$ na temperatura da superfície global no período de 2016 a 2035 em relação a 1986 a 2005 (1). Entre outras conclusões, o relatório apontou que, embora os problemas de saúde humana decorrentes das alterações climáticas sejam considerados relativamente pequenos em comparação com
Fundação Oswaldo Cruz (Fiocruz), Instituto de Comunicação e Informação Científica e Tecnológica em Saúde (ICICT), Rio de Janeiro (RJ), Brasil. 
outros fatores, esses efeitos não têm sido claramente quantificados (1).

O clima pode favorecer a criação de ambientes propícios à proliferação de vetores de doenças e também gerar doenças e agravos diretos em virtude de fenômenos climáticos, como inundações (2). Embora haja evidências da influência do clima na ocorrência de algumas doenças (3), ainda não se conhecem todas as doenças sensíveis ao clima (DSC). Além disso, é necessário compreender os mecanismos de exposição, ou seja, quais variáveis climáticas podem influenciar a incidência de doenças e como esse processo se dá. Essas questões têm sido objeto de muitos estudos e suas respostas permitem definir prioridades em pesquisa, políticas públicas e ações de vigilância em saúde voltadas ao monitoramento e controle dos impactos relacionados a altas temperaturas.

Nesse contexto, o objetivo da presente revisão sistemática foi investigar o conhecimen to existente acerca das DSC e dos principais impactos da variação climática sobre a saúde. Além de investigar os estudos sobre DSC em todo o mundo, foi realizado um recorte específico para avaliar os estudos que tiveram o Brasil como objeto.

\section{MATERIAIS E MÉTODOS}

A revisão sistemática foi conduzida conforme a metodologia PRISMA (4). As buscas foram realizadas nas bases LILACS, PubMed, Scopus e SciELO em julho de 2017, sem restrição temporal. As buscas foram realizadas em inglês, espanhol e português; em todas as bases, os termos de busca utilizados foram (climate) AND (disease) AND (sensitive).

Os critérios de inclusão de artigos na revisão sistemática foram: 1) estudar a associação de um desfecho ou agravo a uma ou mais variável climática; e 2) explicitar o desfecho estudado, ou seja, indicar claramente qual doença foi considerada como variável resposta. Foram excluídos estudos referentes a zoologia, botânica, ecologia, clínica médica e bacteriologia; artigos de ecologia de vetores; estudos de desastres (inundação); relatórios técnicos e literatura cinza; artigos cujo desfechos não eram doenças, por exemplo, suicídio; artigos de revisão; e estudos que não explicitavam a variável climática em análise.

Após a realização da busca nas bases mencionadas, as duplicidades foram excluídas. Posteriormente, os artigos foram incluídos na revisão a partir da leitura dos resumos. Quando a leitura dos resumos não foi suficiente, os artigos foram lidos na íntegra.

As informações obtidas dos artigos foram: autores; ano de publicação; país de origem do primeiro autor; local de estudo; metodologia adotada; doença estudada; e variáveis climáticas consideradas na análise. A fim de comparar os locais estudados no mundo, os estudos realizados em nível municipal, estadual ou distrital foram registrados conforme o país estudado.

Para análise da associação entre as principais DSC identificadas e as variáveis climáticas, foi estimado o coeficiente de clusterização (clustering coefficient), de acordo com a teoria dos grafos (5). Desse modo, os desfechos e as variáveis climáticas analisados foram considerados como nós (ou vértices), e cada ligação entre desfechos e variável climática foi chamada de aresta. O coeficiente de clusterização consiste na divisão do número efetivo de arestas que cada nó possui pela a somatória de todas as arestas possíveis (6), de acordo com a fórmula:

$$
\mathrm{C}=\frac{<\mathrm{k}>}{\mathrm{N}}
$$

onde $<\mathrm{k}>$ é o número de arestas de cada nó $i$ e $N$ é o número total de arestas possíveis, ou seja, número de ligações entre DSC e variáveis climáticas. O gráfico apresentado foi gerado através do Excel, versão 2010, e o mapa foi elaborado usando o Tableau Public Desktop, versão 9.1 .

\section{RESULTADOS}

Através da busca inicial foram identificadas 1162 publicações. Após retirada de 145 duplicatas e exclusão de 911 artigos, permaneceram 106 publicações (7-112) (figura 1).

No total foram identificadas 65 DCS (tabela 1). As DCS mais frequentes foram as doenças respiratórias $(\mathrm{n}=24)$, seguidas por dengue $(n=20)$, malária $(n=18)$, doenças cardiovasculares $(n=16)$, arboviroses $(n=15)$, doenças do sistema digestivo $(n=12)$, doenças bacterianas $(n=10)$ e doenças parasitárias $(n=7)$. Todos as outras DCS foram mencionadas, no máximo, cinco vezes. Para fins de análise, os desfechos foram agrupados, sempre que possível, nas seguintes categorias de doenças: respiratórias, cardiovasculares, do sistema digestivo, parasitárias, zoonoses e transmitidas por vetores, exceto dengue e malária. Tanto dengue como malária foram analisadas de forma individualizada, devido ao grande número de estudos dedicados a essas duas doenças.

FIGURA 1. Fluxo de seleção de artigos na revisão sistemática sobre doenças sensíveis ao clima (DSC)




TABELA 1. Variáveis climáticas associadas às doenças sensíveis ao clima identificadas na revisão sistemática e estudos correspondentes

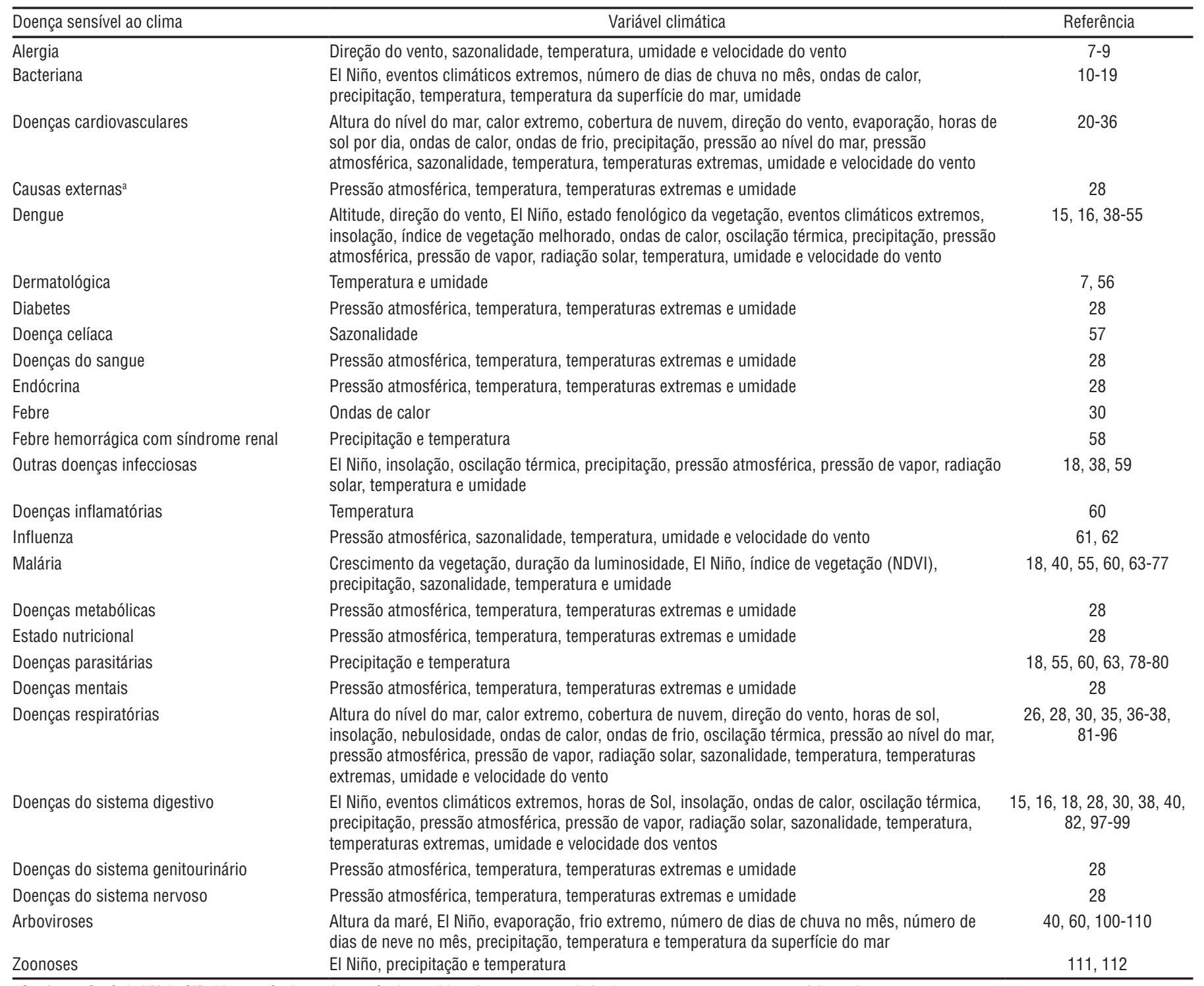

a Conforme Capítulo XX da CID-10: ocorrências e circunstâncias ambientais como causa de lesões, envenenamento e outros efeitos adversos.

Em relação às variáveis climáticas, foram mencionadas 35 variáveis (tabela 1 ). A temperatura foi a mais estudada, em 81 estudos, seguida por precipitação em 50 estudos, umidade em 29, El Niño/ La Niña em 13 e sazonalidade em 12. Todas as outras variáveis climáticas foram mencionadas em menos de 10 publicações.

A figura 2 apresenta a associação entre variáveis climáticas e as DSC mais frequentes. Para essa análise, as DSC foram agrupadas em cinco categorias. O número de variáveis climáticas estudadas em relação a cada categoria de DSC foi dado pelo coeficiente de clusterização (C). De acordo com a teoria de grafos (5), as variáveis climáticas e os desfechos apresentados na figura 2 podem ser considerados vértices ou nós, enquanto as conexões entre eles são chamadas de arestas. Desse modo, foram apresentados cinco nós de categorias de DSC associados a 33 nós de variáveis climáticas. Assim, cada categoria de DSC poderia estar ligada a, no máximo, 33 variáveis climáticas. As doenças respiratórias $(C=0,57)$, cardiovasculares $(C=0,48)$ e a dengue $(C=0,48)$ foram associadas a um número maior de variáveis climáticas, enquanto a malária e outras arboviroses tiveram sua incidência associada a um número menor de variáveis climáticas $(C=0,24$ e $C=0,27)$.

Observando somente as variáveis climáticas, é possível identificar que sete entre 33 foram associadas a somente uma categoria de DSC: altitude e eventos climáticos extremos foram associados estritamente a dengue; crescimento da vegetação a malária; nebulosidade a doenças respiratórias; e número de dias ou de meses de chuva ou neve, temperatura da superfície do mar e altura da maré se associaram às demais arboviroses. 
FIGURA 2. Doenças sensíveis ao clima com maior número de publicações e associação com variáveis climáticas

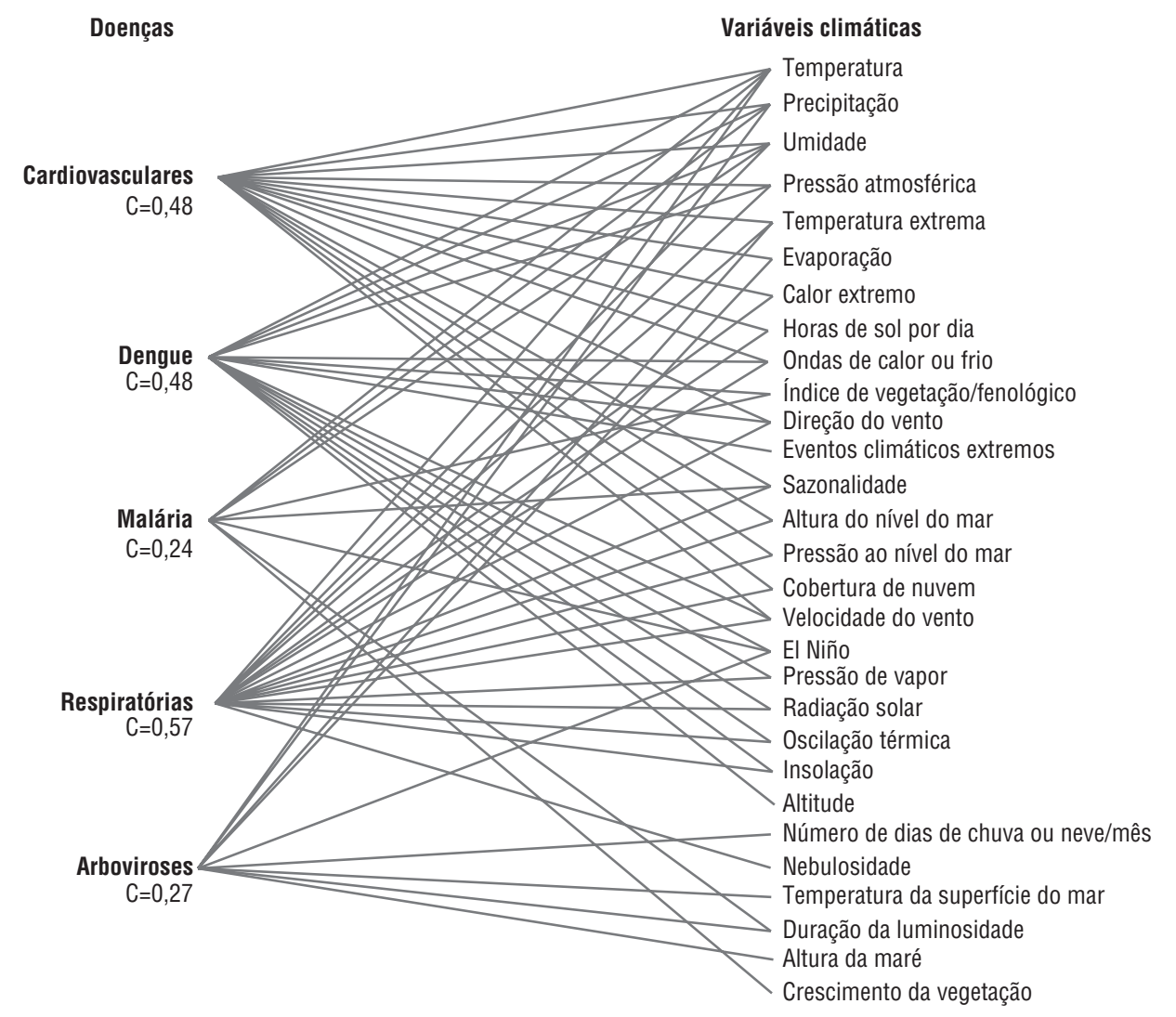

${ }^{a} \mathrm{C}=$ coeficiente de clusterização. Quanto maior o $\mathrm{C}$, maior o número de variáveis climáticas associadas.

\section{Local de estudo e origem dos autores}

Em relação aos locais estudados, foram identificadas 37 unidades geográficas. Apenas um estudo não indicou um local específico (73). A escala de análise foi o mundo em sete publicações $(39,46$, 55, 63-65, 71). As principais unidades geográficas de estudo foram China, com 14 estudos $(18,20,27,32,33,35,40,58,61$, $69,80,91,98,99)$, Estados Unidos, com 12 $(8,36,41,56,87,88,89,90,92,95,97,18)$, Austrália, com nove (17, 19, 22, 31, 81, 103-106), Brasil, com oito $(13,29,44,45$, 47, 51, 54, 87), África, com sete (14, 66, 67, $72,75,77,79)$, Colômbia, com três $(43,49$, 52) e Costa Rica, com três estudos (48, 101, 102).

As DSC variaram de acordo com os locais de estudo, predominando doenças transmitidas por vetores, principalmente malária e dengue, e outras doenças infecciosas, em países tropicais localizados na América Latina, África e Oceania. Os países desenvolvidos onde esses desfechos foram estudados foram
Austrália, Estados Unidos e Suécia. Além desses, foi identificada a China como local de estudo desses desfechos. Já as doenças cardiovasculares e respiratórias apresentaram maior número de estudos em locais temperados e desenvolvidos, principalmente a Europa (23, $24,33,36,37,83,85,88,89,90,92-96)$.

Como esperado, o número de locais de origem dos primeiros autores não coincide com o número de locais estudados (número menor de locais estudados). Foram identificados 32 países de origem do primeiro autor. Entretanto, foi verificada alta concentração - $68 \%$ dos locais de origem dos autores estavam distribuídos em apenas sete países: Estados Unidos $(n=23)$, China $(n=13)$, Reino Unido $(\mathrm{n}=12)$, Austrália $(\mathrm{n}=11)$, Brasil $(\mathrm{n}=8)$, Holanda $(n=4)$ e Suécia $(n=3)$.

\section{Abordagem metodológica dos artigos}

As publicações foram analisadas quanto ao método empregado - análise retrospectiva (séries históricas) ou prospectiva (modelagem para estimar a projeção futura de doenças em associação a diferentes cenários climáticos). Entre os 106 estudos identificados, somente três não puderam ser classificados desse modo, sendo um baseado em dados experimentais (24), um observacional (9) e outro que utilizou dados primários (43).

A figura 3 apresenta a distribuição temporal dos artigos desde o ano de publicação do artigo mais antigo incluído na revisão (ano de 1976) (24), assim como a distribuição temporal dos artigos prospectivos e retrospectivos. Os dois artigos incluídos na revisão e publicados em 2017 não são apresentados na figura 3 , pois as buscas nesse ano se referiram apenas aos 6 primeiros meses. Apesar de o artigo mais antigo ter data de 1976, somente a partir de 1995 foi identificada ao menos uma publicação por ano. Em 2006, houve um salto no número de publicações (nove publicações).

\section{Mudanças climáticas}

A preocupação com as mudanças climáticas atribuídas à emissão de GEE 
FIGURA 3. Número de artigos referentes às doenças sensíveis ao clima publicados entre 1976 e 2016 e número de estudos de acordo com a metodologia adotada (retrospectivos e prospectivos)

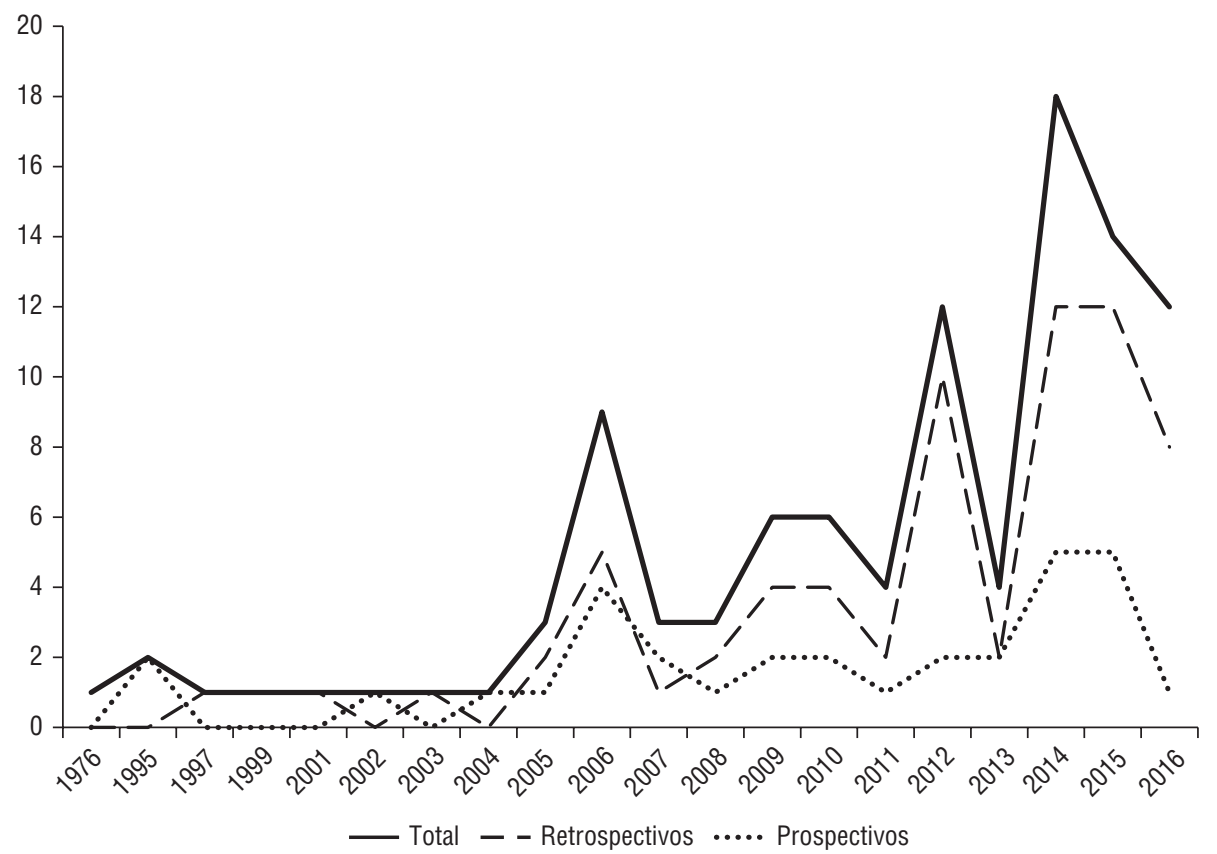

esteve presente em $61 \%$ das publicações. Dentre os estudos prospectivos, $84 \%$ mencionaram as mudanças climáticas, enquanto nos estudos retrospectivos somente $50 \%$ fizeram essa menção. Menos de $15 \%$ dos artigos utilizaram cenários de mudança climática para projeção de impactos à saúde. Todos os estudos que realizaram projeções de incidência de doenças segundo diferentes cenários climáticos utilizaram os cenários e os modelos globais propostos pelo IPCC (1).

\section{Estudos realizados no Brasil}

Foram identificados oito estudos realizados no Brasil, nos anos de 2006 (51), 2008 (44), 2009 (29), 2011 (47) e 2012 (13, $45,54,87)$. Nos estudos brasileiros se manteve o padrão apresentado nos estudos internacionais, com a temperatura sendo a principal variável climática estudada (8), seguida por precipitação (7) e umidade (3). A velocidade do vento surgiu em dois estudos $(51,87)$, e as demais variáveis foram mencionadas somente uma vez: sazonalidade (29), número de dias com chuva no mês (13), direção do vento (51), pressão atmosférica (51) e El Niño (54). Assim como os estudos internacionais, os desfechos foram analisados considerando mais de uma variável climática. Um único estudo, cujo desfecho foram as doenças cardiovasculares, contemplou somente uma variável climática (sazonalidade) (29).

A dengue foi o principal desfecho de análise, sendo contemplado por cinco dos oito estudos $(44,45,47,51,54)$. Os outros desfechos de análise foram: leptospirose (13), doenças cardiovasculares (29) e doenças respiratórias (87). Exceto por dois artigos $(47,54)$, publicados por um mesmo autor do Reino Unido, todos os estudos realizados no Brasil tiveram como primeiro autor um pesquisador brasileiro.

\section{DISCUSSÃO}

Foi realizada uma revisão sistemática para investigar o conhecimento acerca das DSC e de sua associação com variáveis climáticas no Brasil e no mundo. Dentre as doenças identificadas, se destacaram aquelas comumente associadas ao clima, como as arboviroses e as doenças respiratórias. Também houve destaque para doenças com grande incidência mundial, como as doenças cardiovasculares.

A presença frequente das doenças transmitidas por vetores nos estudos selecionados reflete a forte dependência desses vetores das condições climáticas. O ciclo de vida dos vetores, os reservatórios e os hospedeiros estão diretamente relacionados à dinâmica dos ecossistemas onde vivem e, consequentemente, às variáveis climáticas (3).

A dengue é considerada a principal doença reemergente nos países tropicais e subtropicais (113); enquanto a malária é ainda a nona causa de óbitos no mundo e continua sendo um dos maiores problemas de saúde pública na África subsaariana, no sudeste asiático e nos países amazônicos (113). Especificamente no caso brasileiro, a ocorrência endêmica da malária tem se limitado, desde a década de 9160, à região amazônica, embora com registro de alguns casos em outras regiões (114). Embora os casos de malária apresentem redução no Brasil desde as décadas de 1990 e 2000, há necessidade do contínuo controle dessa e de outras arboviroses, além do estudo da sua incidência em associação com fatores ambientais, visto o agravamento de atividades de forte impacto ambiental na região amazônica, como intensificação do desmatamento e implantação de grandes empreendimentos, como projetos hidrelétricos $(114,115)$.

Além da dengue e da malária, outras arboviroses foram identificadas, com importância sanitária variada, em todos os continentes (3), principalmente em regiões da África, Ásia e América Latina. A leishmaniose tegumentar americana, também transmitida por vetor, foi mencionada em cinco publicações e apresenta reconhecida associação com fatores ambientais e climáticos (116).

As doenças cardiovasculares apresentam maior carga global nos países desenvolvidos e em muitos países em desenvolvimento. De acordo com o estudo da Carga Global de Doenças de 2015 (117), as três principais causas de morte em 2015 no mundo foram doença isquêmica do coração, doenças cerebrovasculares e infecções do sistema respiratório inferior. Esses resultados corroboram a crescente preocupação com doenças cardiovasculares em situações de adversidade climática, como ondas de calor e temperaturas extremas, além do aumento da temperatura média.

Os resultados referentes à clusterização permitiram analisar a diversidade de variáveis climáticas que têm sido abordadas em relação às principais categorias de DSC estudadas. As doenças respiratórias e cardiovasculares e a dengue apresentaram altos coeficientes de clusterização, indicando que estiveram associadas a um maior número de variáveis climáticas. Já a malária e demais 
arboviroses têm sido associadas a uma diversidade menor de variáveis climáticas. Além da análise dos desfechos, a figura 2 e a tabela 1 permitem identificar variáveis climáticas pouco estudadas em relação à saúde humana, como nebulosidade e número total de dias de chuva ou neve.

As principais variáveis climáticas consideradas na investigação da interferência do clima sobre os desfechos foram temperatura e precipitação. Isso se deve, provavelmente, à facilidade de acesso a esses dados e ao monitoramento dessas variáveis por longos períodos, além da presença desses parâmetros nos cenários climáticos propostos pelo IPCC (1). Outras variáveis consideradas nos estudos apresentam menor disponibilidade de acesso e padronização para comparação internacional, como índices de cobertura vegetal, número de dias de chuva ou de neve ao mês, número de dias de estresse térmico e ondas de calor.

O número de estudos referentes ao Brasil foi inferior ao esperado, possivelmente em virtude das palavras-chave utilizadas, que restringiram a obtenção de artigos. A expectativa de obtenção de um número maior de artigos se deve à presença de diversos grupos de pesquisa no país, representados principalmente pela Rede Clima (redeclima.ccst.inpe.br) e pelo Observatório Saúde e Clima (climasaude.icict.fiocruz.br), que congregam diversas instituições de pesquisa e universidades.

Os estudos brasileiros obtidos enfocaram principalmente a dengue. Esse resultado ilustra a preocupação resultante dos recorrentes surtos epidêmicos registrados no país a partir de 2005, com identificação das quatro variações do vírus da dengue circulantes no país (118).

Analisando os locais de estudo, é possível verificar que os impactos sobre os sistemas humanos não ocorrem com distribuição geográfica homogênea, devido aos diferentes resultados previstos pelas mudanças climáticas, além de distintas características socioeconômicas (1). A heterogeneidade espacial é verificada na distribuição dos locais de estudo, com maior concentração dos estudos de doenças infecciosas e de arboviroses nas regiões tropicais, enquanto as doenças respiratórias e cardiovasculares têm suas pesquisas melhor distribuídas.

Diferentes ações e acordos internacionais têm sido promovidos, visando à mitigação dos impactos das mudanças climáticas. Dentre as últimas ações realizadas, destaca-se a $21^{\text {a }}$ Conferência das Partes da Convenção Quadro das Nações Unidas sobre Mudanças do Clima (COP21), realizada em dezembro de 2015. Ao final dessa convenção, foi aprovado o Acordo de Paris, com 195 países participantes. Esse encontro foi considerado um marco histórico no processo de combate às mudanças climáticas globais para muitos atores envolvidos no processo. Contudo, suas metas foram consideradas tímidas por uma parcela da comunidade científica (2). O objetivo principal desse acordo, que entra em vigência em 2020, é manter o aumento da temperatura abaixo de $2^{\circ} \mathrm{C}$, procurando alcançar a meta de $1,5^{\circ} \mathrm{C}$.

As mudanças na política internacional no período após a assinatura do acordo, como a postura anticonservacionista do presidente dos Estados Unidos eleito em 2016 e a quebra da unidade da União Europeia, podem comprometer as metas acordadas, ainda que sejam consideradas insuficientes.

Além do IPCC e da COP21, destaca-se a menção direta às mudanças climáticas no Objetivo 13 dos Objetivos de Desenvolvimento Sustentável (ODS) no que se refere a "tomar medidas urgentes para combater a mudança do clima e os seus impactos" (119). Outros ODS têm relação com a ocorrência das DSC, pois tratam da promoção de ações e ambientes sustentáveis, assim como de melhorias nas condições sanitárias.

Especificamente em relação ao Brasil, concluiu-se, em setembro de 2016, o processo de ratificação do Acordo de Paris após aprovação pelo Congresso Nacional, quando as metas brasileiras passaram a ser compromissos oficiais (120, 121). Desde então, o Brasil comprometese a reduzir as emissões de GEE em relação às taxas de 2005, em 37\% até 2015 e $43 \%$ até 2030.

Embora esta revisão sistemática apresente as DSC estudadas no Brasil e no mundo, a estratégia de busca utilizada apresentou algumas limitações, visto que muitos estudos não utilizam a expressão "doença sensível ao clima". Por outro lado, a menção à "doença sensível ao clima", quando esteve presente, demonstrou uma preocupação dos autores em enfatizar as possíveis relações entre clima e saúde e o impacto das mudanças climáticas sobre a saúde.

Outra limitação do estudo foi a delimitação temporal, pois a busca de artigos não contemplou todo o ano de 2017, o que pode ter resultado na ausência de estudos sobre Zika e chikungunya, visto que essas doenças tiveram dispersão mundial recente. A busca estritamente em repositórios de artigos também impediu a obtenção de estudos publicados em conferências e literatura cinza.

O grande número de artigos selecionados inviabilizou a avaliação dos artigos conforme todas as orientações da PRISMA. Muitas das publicações selecionadas podem ter adotado métodos questionáveis ou de baixa qualidade. As limitações metodológicas dos artigos selecionados podem ter resultado na inclusão de variáveis climáticas e doenças que não estejam necessariamente associadas. A ausência da avaliação da qualidade dos artigos também impediu a identificação de variáveis de confusão, tanto no que se refere às variáveis climáticas como às doenças sensíveis ao clima.

Apesar das limitações, os resultados obtidos por esta revisão sistemática fornecem um panorama atual do conhecimento sobre as doenças sensíveis ao clima, principais desfechos sobre a saúde e sua associação com variáveis climáticas, no Brasil e no mundo. Os resultados obtidos indicaram grande concentração de variáveis climáticas estudadas, sugerindo a necessidade de fomentar $\mathrm{O}$ registro e a disponibilidade de dados referentes a outras variáveis, além de temperatura e precipitação. Também foi verificada a necessidade de ampliar os países estudados, considerando os impactos previstos pelas mudanças climáticas, assim como a diversidade ambiental, climática e socioeconômica que incidem de maneira diversa sobre as doenças sensíveis ao clima.

Agradecimentos. TCMS recebeu financiamento do Instituto de Pesquisa em Econômica Aplicada (IPEA - 093/2014). CB recebeu financiamento do Conselho Nacional de Desenvolvimento Científico e Tecnológico (CNPq - 404160/2013-1).

Conflitos de interesse. Nada declarado pelos autores.

Declaração. As opiniões expressas no manuscrito são de responsabilidade exclusiva dos autores e não refletem necessariamente a opinião ou políticas da RPSP/PAJPH ou da Organização PanAmericana da Saúde (OPAS). 


\section{REFERÊNCIAS}

1. Pachauri RK, Mayer L. Intergovernmental Panel on Climate Change. Climate change 2014: synthesis report. Genebra: Intergovernmental Panel on Climate Change; 2015. Disponível em: https:// www.weadapt.org/knowledge-base / national-adaptation-planning/climatechange-2014-synthesis-report Acessado em abril de 2018.

2. Barcellos C, Hacon S de S. Um grau e meio. E daí? Cad Saude Publica. 2016; 32(3):e00212315.

3. Barcellos C, Monteiro AMV, Corvalán C, Gurgel HC, Carvalho MS, Artaxo P, et al. Mudanças climáticas e ambientais e as doenças infecciosas: cenários e incertezas para o Brasil. Epidemiol Serv Saude. 2009;18(3):285-304.

4. Moher D, Liberati A, Tetzlaff J, Altman DG, PRISMA Group. Preferred reporting items for systematic reviews and metaanalyses: the PRISMA statement. PLoS Med. 2009;6(7):e1000097.

5. Barabasi AL. Linked: the new science of networks. Cambridge: Perseus Publishing; 2002.

6. Albert R, Barabasi AL. Statistical mechanics of complex networks. Rev Mod Phys. 2002;74(1):47-97.

7. Hamadeh RR, Al-Roomi KA. Air quality and seasonal variations in consultations for respiratory, allergic, dermatological and gastrointestinal diseases in Bahrain, 2007. East Mediterr Health J. 2014;20(5):309-16.

8. Zhang R, Duhl T, Salam MT, House JM, Flagan RC, Avol EL, et al. Development of a regional-scale pollen emission and transport modeling framework for investigating the impact of climate change on allergic airway disease. Biogeosciences. 2014;11(6):1461-78.

9. Blanca-Lopez N, Campo P, Salas M, García Rodríguez C, Palomares F, Blanca M, et al. Seasonal local allergic rhinitis in areas with high concentrations of grass pollen. J Investig Allergol Clin Immunol. 2016; 26(2):83-91.

10. Alajo SO, Nakavuma J, Erume J. Cholera in endemic districts in Uganda during El Niño rains: 2002-2003. Afr Health Sci. 2006;6(2):93-7.

11. Reiner RC, King AA, Emch M, Yunus M, Faruque ASG, Pascual M. Highly localized sensitivity to climate forcing drives endemic cholera in a megacity. Proc Natl Acad Sci. 2012;109(6):2033-6.

12. Leckebusch GC, Abdussalam AF. Climate and socioeconomic influences on interannual variability of cholera in Nigeria. Health Place. 2015;34:107-17.

13. Oliveira TV, Marinho DP, Costa Neto C, Kligerman DC. Climate variables, living conditions and the health of the population: leptospirosis in the city of Rio de Janeiro from 1996 to 2009. Cienc Saude Coletiva. 2012;17(6):1569-76.

14. Paz S. Impact of temperature variability on cholera incidence in Southeastern Africa, 1971-2006. EcoHealth. 2009;6(3):340-5.

15. McIver L, Hashizume M, Kim H, Honda $Y$, Pretrick M, Iddings S, et al. Assessment of climate-sensitive infectious diseases in the Federated States of Micronesia. Trop Med Health. 2015;43(1):29-40.

16. McIver L, Kim R, Woodward A, Hales S, Spickett J, Katscherian D, et al. Health impacts of climate change in Pacific Island countries: a regional assessment of vulnerabilities and adaptation priorities. Environ Health Perspect. 2015;124(11): 1707-14.

17. Milazzo A, Giles LC, Zhang Y, Koehler AP, Hiller JE, Bi P. Heatwaves differentially affect risk of Salmonella serotypes. J Infect. 2016;73(3):231-40.

18. Wang Y, Rao Y, Wu X, Zhao H, Chen J. A method for screening climate change-sensitive infectious diseases. Int J Environ Res Public Health. 2015;12(1):767-83.

19. Milazzo A, Giles LC, Zhang Y, Koehler AP, Hiller JE, Bi P. The effect of temperature on different Salmonella serotypes during warm seasons in a Mediterranean climate city, Adelaide, Australia. Epidemiol Infect. 2016;144(6):1231-40.

20. Tian Z, Li S, Zhang J, Jaakkola JJ, Guo Y. Ambient temperature and coronary heart disease mortality in Beijing, China: a time series study. Environ Health. 2012;11(1):56.

21. Chen VY-J, Wu P-C, Yang T-C, Su H-J. Examining non-stationary effects of social determinants on cardiovascular mortality after cold surges in Taiwan. Sci Total Environ. 2010;408(9):2042-9.

22. Webb L, Bambrick H, Tait P, Green D, Alexander L. Effect of ambient temperature on Australian Northern Territory public hospital admissions for cardiovascular disease among indigenous and nonindigenous populations. Int J Environ Res Public Health. 2014;11(2):1942-59.

23. Alberini A, Chiabai A. Urban environmental health and sensitive populations: how much are the Italians willing to pay to reduce their risks? Reg Sci Urban Econom. 2007;37(2):239-58.

24. Andersen I, Jensen PL, Junker P, Thomsen A, Wyon DP. The effects of moderate heat stress on patients with ischemic heart disease. Scand J Work Environ Health. 1976;2(4):256-68.

25. Giang PN, Dung DV, Giang KB, Vinhc HV, Rocklöv J. The effect of temperature on cardiovascular disease hospital admissions among elderly people in Thai Nguyen Province, Vietnam. Glob Health Action. 2014;7(1):23649.

26. Grass D, Cane M. The effects of weather and air pollution on cardiovascular and respiratory mortality in Santiago, Chile, during the winters of 1988-1996. Int J Climatol. 2008;28(8):1113-26.

27. Huang J, Wang J, Yu W. The lag effects and vulnerabilities of temperature effects on cardiovascular disease mortality in a subtropical climate zone in China. Int J Environ Res Public Health. 2014;11(4): 3982-94.

28. Kim CT, Lim Y-H, Woodward A, Kim H. Heat-attributable deaths between 1992 and 2009 in Seoul, South Korea. PLoS One. 2015;10(2):e0118577.
29. Kleinfelder D, Andrade JL, Schlaad SW, Carvalho FC, Bellen BV. Seasonal variation of venous thromboembolism in the subtropical climate of São Paulo, Brazil. J Vasc Bras. 2009;8(1):29-32.

30. Leonardi G, Hajat S, Kovats R, Smith G, Cooper D, Gerard E. Syndromic surveillance use to detect the early effects of hea$\mathrm{t}$-waves: an analysis of NHS Direct data in England. Soz Präventivmedizin SPM. 2006;51(4):194-201.

31. Loughnan ME, Nicholls N, Tapper NJ. The effects of summer temperature, age and socioeconomic circumstance on acute myocardial infarction admissions in Melbourne, Australia. Int J Health Geogr. 2010;9(1):41

32. Tian Z, Li S, Zhang J, Guo Y. The characteristic of heat wave effects on coronary heart disease mortality in Beijing, China: A time series study. PLoS One. 2013;30; 8(9):e77321.

33. Zhang Y, Li S, Pan X, Tong S, Jaakkola JJ, Gasparrini A, et al. The effects of ambient temperature on cerebrovascular mortality: an epidemiologic study in four climatic zones in China. Environ Health. 2014; 13(1):24 .

34. Shaposhnikov D, Revich B, Gurfinkel Y, Naumova E. The influence of meteorological and geomagnetic factors on acute myocardial infarction and brain stroke in Moscow, Russia. Int J Biometeorol. 2014;58(5):799-808.

35. Li T, Ban J, Horton RM, Bader DA, Huang $\mathrm{G}$, Sun $\mathrm{Q}$, et al. Heat-related mortality projections for cardiovascular and respiratory disease under the changing climate in Beijing, China. Sci Rep. 2015;5(1).

36. McDonald YJ, Grineski SE, Collins TW, Kim YA. A scalable climate health justice assessment model. Soc Sci Med. 2015;133:242-52.

37. Smith S, Elliot A, Hajat S, Bone A, Bates C, Smith G, et al. The impact of heatwaves on community morbidity and healthcare usage: a retrospective observational study using real-time syndromic surveillance. Int J Environ Res Public Health. 2016;13(1):132.

38. Ortíz Bultó PL, Rodríguez AP, Valencia AR, Vega NL, Díaz M, Carrera AP. Assessment of human health vulnerability to climate variability and change in Cuba. Environ Health Perspect. 2006;114(12): 1942-49.

39. Hales S, de Wet N, Maindonald J, Woodward A. Potential effect of population and climate changes on global distribution of dengue fever: an empirical model. The Lancet. 2002;360(9336): 830-4.

40. Hodges M, Belle JH, Carlton EJ, Liang S, Li $\mathrm{H}$, Luo W, et al. Delays in reducing waterborne and water-related infectious diseases in China under climate change. Nat Clim Change. 2014;4(12):1109-15.

41. Kolivras K. Changes in dengue risk potential in Hawaii, USA, due to climate variability and change. Clim Res. 2010; 42(1):1-11. 
42. Xuan LTT, Van Hau P, Thu DT, Toan DTT. Estimates of meteorological variability in association with dengue cases in a coastal city in northern Vietnam: an ecological study. Glob Health Action. 2014;7(1):23119.

43. Azoh Barry J. Dengue threat: adaptation needs in a disadvantaged neighborhood in Medellín-Colombia. Rev Costarric Salud Publica. 2011;20(1):16-24.

44. Dibo MR, Chierotti AP, Ferrari MS, Mendonça AL, Chiaravalloti Neto F. Study of the relationship between Aedes (Stegomyia) aegypti egg and adult densities, dengue fever and climate in Mirassol, state of São Paulo, Brazil. Mem Inst Oswaldo Cruz. 2008;103(6):554-560.

45. Gomes AF, Nobre AA, Cruz OG. Temporal analysis of the relationship between dengue and meteorological variables in the city of Rio de Janeiro, Brazil, 2001-2009. Cad Saude Publica. 2012;28(11):2189-2197.

46. Liu-Helmersson J, Stenlund H, WilderSmith A, Rocklöv J. Vectorial Capacity of Aedes aegypti: Effects of temperature and implications for global dengue epidemic potential. PLoS One. 2014;9(3):e89783.

47. Lowe R, Bailey TC, Stephenson DB, Graham RJ, Coelho CAS, Sá Carvalho M, et al. Spatio-temporal modelling of climate-sensitive disease risk: Towards an early warning system for dengue in Brazil. Comput Geosci. 2011;37(3):371-81.

48. Mena N, Troyo A, Bonilla-Carrión R, Calderón-Arguedas Ó. Factors associated with incidence of dengue in Costa Rica. Rev Panam Salud Publica. 2011;29(4):234-42.

49. Meza-Ballesta A, Gónima L. The influence of climate and vegetation cover on the occurrence of dengue cases (2001-2010). Rev Salud Publica. 2014;16(2):293-306.

50. Rifakis P, Gonçalves N, Omaña W, Manso M, Espidel A, Intingaro A, et al. Asociación entre las variaciones climáticas y los casos de dengue en un hospital de Caracas, Venezuela, 1998-2004. Rev Peru Med Exp Salud Publica. 2005;22(3):183-90.

51. Rosa-Freitas MG, Schreiber KV, Tsouris $P$, Weimann ET de S, Luitgards-Moura JF. Associations between dengue and combinations of weather factors in a city in the Brazilian Amazon. Rev Panam Salud Publica. 2006;20(4):256-267.

52. Rúa-Uribe GL, Suárez-Acosta C, Chauca J, Ventosilla P, Almanza R. Modelización del efecto de la variabilidad climática local sobre la transmisión de dengue en Medellín (Colombia) mediante análisis de series temporales. Biomedica. 2012;33(Supl. 1):142-52.

53. Lowe R, Cazelles B, Paul R, Rodó X. Quantifying the added value of climate information in a spatio-temporal dengue model. Stoch Environ Res Risk Assess. 2016;30(8):2067-78.

54. Lowe R, Bailey TC, Stephenson DB, Jupp TE, Graham RJ, Barcellos C, et al. The development of an early warning system for climate-sensitive disease risk with a focus on dengue epidemics in Southeast Brazil. Stat Med. 2013;32(5):864-83.

55. Martens WJ, Jetten TH, Focks DA. Sensitivity of malaria, schistosomiasis and dengue to global warming. Clim Change. 1997;35(2):145-56.
56. Kathuria P, Silverberg JI. Association of pollution and climate with atopic eczema in US children. Pediatr Allergy Immunol. 2016;27(5):478-85.

57. Ivarsson A, Hernell O, Nyström L, Persson LA. Children born in the summer have increased risk for coeliac disease. J Epidemiol Community Health. 2003;57(1):36-9.

58. Liu J, Xue FZ, Wang JZ, Liu QY. Association of haemorrhagic fever with renal syndrome and weather factors in Junan County, China: a case-crossover study. Epidemiol Infect. 2013;141(4):697-705.

59. Águila AD, Briceño M. Análisis de la epidemia de muertes infantiles en Talara durante el Fenómeno El Niño de 1997-1998: ¿estamos preparados para enfrentar otra? An Fac Med Lima. 2007;68(2):193-202.

60. Casimiro E, Calheiros J, Santos FD, Kovats S. National assessment of human health impacts of climate change in Portugal: approach and key findings. Environ Health Perspect. 2006;114(12):1950-6.

61. Xiao H, Tian H, Lin X, Gao L, Dai X, Zhang $X$, et al. Influence of extreme weather and meteorological anomalies on outbreaks of influenza A (H1N1). Chin Sci Bull. 2013; 58(7):741-9.

62. Singh BK, Savill NJ, Ferguson NM, Robertson C, Woolhouse ME. Rapid detection of pandemic influenza in the presence of seasonal influenza. BMC Public Health. 2010;10(1):726.

63. Martens WJM, Jetten TH, Rotmans J, Niessen LW. Climate change and vectorborne diseases: a global modelling perspective. Glob Environ Change. 1995;5(3):195-209.

64. van Lieshout $M$, Kovats RS, Livermore MTJ, Martens P. Climate change and malaria: analysis of the SRES climate and socio-economic scenarios. Glob Environ Change. 2004;14(1):87-99.

65. Caminade C, Kovats S, Rocklov J, Tompkins AM, Morse AP, Colón-González FJ, et al. Impact of climate change on global malaria distribution. Proc Natl Acad Sci. 2014;111(9):3286-91.

66. Chaves LF, Satake A, Hashizume M, Minakawa N. Indian Ocean dipole and rainfall drive a Moran effect in East Africa malaria transmission. J Infect Dis. 2012;205(12):1885-91.

67. Gemperli A, Sogoba N, Fondjo E, Mabaso M, Bagayoko M, Briet OJT, et al. Mapping malaria transmission in West and Central Africa. Trop Med Int Health. 2006; 11(7):1032-46.

68. Goswami P, Murty US, Mutheneni SR, Krishnan ST. Relative Roles of Weather Variables and Change in Human Population in Malaria: Comparison over Different States of India. PLoS One. 2014;9(6):e99867.

69. Guo C, Yang L, Ou C-Q, Li L, Zhuang Y, Yang J, et al. Malaria incidence from 20052013 and its associations with meteorological factors in Guangdong, China. Malar J. 2015;14(1):116.

70. Hurtado LA, Cáceres L, Chaves LF, Calzada JE. When climate change couples social neglect: malaria dynamics in Panama. Emerg Microbes Infect. 2014;3(4):e27.
71. Martens WJ, Niessen LW, Rotmans J, Jetten TH, McMichael AJ. Potential impact of global climate change on malaria risk. Environ Health Perspect. 1995;103(5):458-464.

72. Mabaso MLH, Kleinschmidt I, Sharp B, Smith T. El Niño Southern Oscillation (ENSO) and annual malaria incidence in Southern Africa. Trans R Soc Trop Med Hyg. 2007;101(4):326-30.

73. Paaijmans KP, Read AF, Thomas MB. Understanding the link between malaria risk and climate. Proc Natl Acad Sci. 2009;106(33):13844-9.

74. Stuckey EM, Stevenson JC, Cooke MK, Owaga C, Marube E, Oando G, et al. Simulation of malaria epidemiology and control in the highlands of western Kenya. Malar J. 2012;11(1):357.

75. Tonnang HE, Kangalawe RY, Yanda PZ. Predicting and mapping malaria under climate change scenarios: the potential redistribution of malaria vectors in Africa. Malar J. 2010;9(1):111.

76. Garg A, Dhiman RC, Bhattacharya S, Shukla PR. Development, Malaria and adaptation to climate change: a case study from India. Environ Manage. 2009;43(5):779-89.

77. Leedale J, Tompkins AM, Caminade C, Jones AE, Nikulin G, Morse AP. Projecting malaria hazard from climate change in eastern Africa using large ensembles to estimate uncertainty. Geospatial Health. 2016;11(1s):102-14.

78. Caminade C, van Dijk J, Baylis M, Williams D. Modelling recent and future climatic suitability for fasciolosis in Europe. Geospatial Health. 2015;9(2):301-8.

79. Moore S, Shrestha S, Tomlinson KW, Vuong H. Predicting the effect of climate change on African trypanosomiasis: integrating epidemiology with parasite and vector biology. $\mathrm{J} R$ Soc Interface. 2012;9(70):817-30.

80. Zhou X-N, Yang G-J, Yang K, Wang X-H, Hong Q-B, Sun L-P, et al. Potential impact of climate change on schistosomiasis transmission in China. Am J Trop Med Hyg. 2008;78(2):188-94.

81. Xu Z, Liu Y, Ma Z, Li S, Hu W, Tong S. Impact of temperature on childhood pneumonia estimated from satellite remote sensing. Environ Res. 2014;132:334-41.

82. Hamadeh RR, Al-Roomi KA. Air quality and seasonal variations in consultations for respiratory, allergic, dermatological and gastrointestinal diseases in Bahrain, 2007. East Mediterr Health J. 2014;20(5):309-16.

83. Heal MR, Heaviside C, Doherty RM, Vieno M, Stevenson DS, Vardoulakis S. Health burdens of surface ozone in the UK for a range of future scenarios. Environ Int. 2013;61:36-44.

84. Matthew J, Pinto Pereira LM, Pappas TE, Swenson CA, Grindle KA, Roberg KA, et al. Distribution and seasonality of rhinovirus and other respiratory viruses in a cross-section of asthmatic children in Trinidad, West Indies. Ital J Pediatr. 2009; 35:16.

85. Lin S, Hsu WH, Van Zutphen AR, Saha S, Luber G, Hwang SA. Excessive heat and respiratory hospitalizations in New York State: 
estimating current and future public health burden related to climate change. Environ Health Perspect. 2012;120(11):1571-7.

86. Pazitková V, Martínez VP, González AA, Estela LL. El asma bronquial y su asociación con los cambios de tiempo. Rev Cuba Med Gen Integral. 2010;26(4):665-672.

87. Souza A de, Fernandes WA, Pavão HG, Lastoria G, Albrez E do A. Potential impacts of climate variability on respiratory morbidity in children, infants, and adults. J Bras Pneumol. 2012;38(6):708-715.

88. Sheffield PE, Zhou J, Shmool JLC, Clougherty JE. Ambient ozone exposure and children's acute asthma in New York City: a case-crossover analysis. Environ Health. 2015;14(1):25.

89. Teach SJ, Gergen PJ, Szefler SJ, Mitchell HE, Calatroni A. Wildfire J, et al. Seasonal risk factors for asthma exacerbations among inner-city children. J Allergy Clin Immunol. 2015;135(6):1465-1473.e5.

90. Wisniewski JA, McLaughlin AP, Stenger PJ, Patrie J, Brown MA, El-Dahr JM, et al. A comparison of seasonal trends in asthma exacerbations among children from geographic regions with different climates. Allergy Asthma Proc. 2016;37(6):475-81.

91. Zhang J, Dai J, Yan L, Fu W, Yi J, Chen Y, et al. Air pollutants, climate, and the prevalence of pediatric asthma in urban areas of China. Biomed Res Int. 2016;2016:2935163.

92. Eggo RM, Scott JG, Galvani AP, Meyers LA. Respiratory virus transmission dynamics determine timing of asthma exacerbation peaks: Evidence from a population-level model. Proc Natl Acad Sci. 2016;113(8):2194-9.

93. Altzibar JM, Tamayo-Uria I, De Castro V, Aginagalde X, Albizu MV, Lertxundi A, et al. Epidemiology of asthma exacerbations and their relation with environmental factors in the Basque Country. Clin Exp Allergy. 2015;45(6):1099-108.

94. Islam MS, Chaussalet TJ, Koizumi N. Towards a threshold climate for emergency lower respiratory hospital admissions. Environ Res. 2017;153:41-7.

95. O'Lenick CR, Winquist A, Chang HH, Kramer MR, Mulholland JA, Grundstein $\mathrm{A}$, et al. Evaluation of individual and area-level factors as modifiers of the association between warm-season temperature and pediatric asthma morbidity in Atlanta, GA. Environ Res. 2017;156:132-44.

96. Sato S, Saito J, Suzuki Y, Uematsu M, Fukuhara A, Togawa R, et al. Association between typhoon and asthma symptoms in Japan. Respir Investig. 2016;54(3):216-9.

97. Jensen ET, Shah ND, Hoffman K, Sonnenberg A, Genta RM, Dellon ES. Seasonal variation in detection of oesophageal eosinophilia and eosinophilic oesophagitis. Aliment Pharmacol Ther. 2015;42(4):461-9.

98. Li K, Zhao K, Shi L, Wen L, Yang H, Cheng $\mathrm{J}$, et al. Daily temperature change in relation to the risk of childhood bacillary dysentery among different age groups and sexes in a temperate city in China. Public Health. 2016;131:20-6.

99. Liu J, Wu X, Li C, Xu B, Hu L, Chen J, et al. Identification of weather variables sensitive to dysentery in disease-affected county of China. Sci Total Environ. 2017;575:956-62.

100. Chaves LF, Calzada JE, Valderrama A, Saldaña A. Cutaneous Leishmaniasis and Sand Fly Fluctuations Are Associated with El Niño in Panamá. PLoS Negl Trop Dis. 2014;8(10):e3210.

101. Chaves LF, Pascual M. Climate Cycles and Forecasts of Cutaneous Leishmaniasis, a Nonstationary VectorBorne Disease. PLoS Med. 2006;3(8):e295.

102. Chaves LF, Pascual M. Comparing models for early warning systems of neglected tropical diseases. PLoS Negl Trop Dis. 2007;1(1):e33.

103. Gatton ML, Kay BH, Ryan PA. Environmental predictors of Ross River virus disease outbreaks in Queensland, Australia. Am J Trop Med Hyg. 2005;72(6): 792-799.

104. Woodruff RE, Guest CS, Garner MG, Becker N, Lindsay M. Early Warning of Ross River Virus Epidemics: Combining Surveillance Data on Climate and Mosquitoes. Epidemiology. 2006;17(5):569-75.

105. Naish S, Mengersen K, Hu W, Tong S. Forecasting the Future Risk of Barmah Forest Virus Disease under Climate Change Scenarios in Queensland, Australia. PLoS One. 2013;8(5):e62843.

106. Maelzer D, Hales S, Weinstein P, Zalucki M, Woodward A. El Nino and arboviral disease prediction. Environ Health Perspect. 1999;107(10):817-8.

107. Shirzadi MR, Mollalo A, YaghoobiErshadi MR. Dynamic relations between incidence of zoonotic cutaneous leishmaniasis and climatic factors in Golestan Province, Iran. J Arthropod Borne Dis. 2015;9(2):148-60.

108. Day JF, Shaman J. Severe Winter Freezes Enhance St. Louis Encephalitis Virus Amplification and Epidemic Transmission in Peninsular Florida. J Med Entomol. 2009;46(6):1498-506.

109. Palo RT. Tick-Borne Encephalitis Transmission Risk: Its Dependence on Host Population Dynamics and Climate Effects. Vector-Borne Zoonotic Dis. 2014; 14(5):346-52.

110. Paz S, Albersheim I. Influence of Warming Tendency on Culex pipiens Population Abundance and on the Probability of West Nile Fever Outbreaks (Israeli Case Study: 2001-2005). EcoHealth. 2008;5(1):40-8.

111. Anyamba A, Linthicum KJ, Tucker CJ. Climate-disease connections: Rift Valley fever in Kenya. Cad Saude Publica. 2001;17Suppl:133-40.

112. Mpeshe SC, Luboobi LS, NkansahGyekye Y. Modeling the Impact of Climate Change on the Dynamics of Rift Valley Fever. Comput Math Methods Med. 2014;2014:627586.
113. Vos T, Allen C, Arora M, Barber RM, Bhutta ZA, Brown A, et al. Global, regional, and national incidence, prevalence, and years lived with disability for $310 \mathrm{di}-$ seases and injuries, 1990-2015: a systematic analysis for the Global Burden of Disease Study 2015. Lancet. 2016; 388(10053):1545-602.

114. Katsuragawa TH, Gil LHS, Tada MS, da Silva LHP. Endemias e epidemias na Amazônia: malária e doenças emergentes em áreas ribeirinhas do Rio Madeira. Um caso de escola. Estud Av. 2008;22(64):111-41.

115. Vasconcelos $\mathrm{CH}$, Novo EML de M, Donalisio MR. Uso do sensoriamento remoto para estudar a influência de alterações ambientais na distribuição da malária na Amazônia brasileira. Cad Saude Publica. 2006;22(3):517-26.

116. Brasil, Ministério da Saúde, Secretaria de Vigilância em saúde, Departamento de vigilância epidemiológica. Doenças infecciosas e parasitárias: guia de bolso Brasília: Ministério da Saúde; 2010. Disponível em: http://bvsms.saude.gov. $\mathrm{br} / \mathrm{bvs} /$ publicacoes/doencas_infecciosas_parasitaria_guia_bolso.pdf Acessado em abril de 2018.

117. Nomura S, Sakamoto H, Glenn S, Tsugawa Y, Abe SK, Rahman MM, et al. Population health and regional variations of disease burden in Japan, 1990-2015: a systematic subnational analysis for the Global Burden of Disease Study 2015. Lancet. 2017;390(10101):1521-1538.

118. Barreto ML, Teixeira MG. Dengue no Brasil: situação epidemiológica e contribuições para uma agenda de pesquisa. Estud Av. 2008;22(64):53-72

119. United Nations (UN). Sustainable Development Goals: 17 goals to transform our world. Disponível em: https: / /www. un.org/sustainabledevelopment / sustainable-development-goals / Acessado em maio de 2018.

120. Gonzalez-Perez MA. Climate Change and the 2030 Corporate Agenda for Sustainable Development. Em: GonzalezPerez MA, Leonard L, eds. Volume 19 Climate Change and the 2030 Corporate Agenda for Sustainable Development. West Yorkshire: Emerald Group Publishing Limited; 2016. Pp. 1-6.

121. Kässmayer K, Neto HJF. A Entrada em Vigor do Acordo de Paris: o que muda para o Brasil? Brasília: Núcleo de Estudos e Pesquis a s / CONLEG / S en a d o, Novembro/2016 (Texto para Discussão n 215). Disponível em: http://www2.senado.leg.br/bdsf/handle/id / 528873 Acessado em 4 de maio de 2018.

Artigo recebido em 8 de setembro de 2017. Aceito em versão revisada em 12 de abril de 2018. 
ABSTRACT Objective. To survey the literature regarding climate-sensitive diseases (CSD) and the impacts of climate changes on health.

Method. This systematic review was conducted according to the Preferred Reporting Items for Systematic Reviews and Meta-Analyses (PRISMA). The Lilacs, SciELO,

\section{Climate-sensitive diseases in Brazil and the world: systematic review}

Keywords Climate change; climate effects; dengue; malaria; respiratory tract diseases; cardiovascular diseases; Brazil.
RESUMEN

\section{Enfermedades sensibles al clima en Brasil y el mundo: revisión sistemática}

Palabras-clave
Objetivos. Hacer un examen de las publicaciones sobre las enfermedades sensibles al clima y los efectos de las alteraciones climáticas sobre la salud

Método. La revisión sistemática se efectuó de conformidad con el método basado en elementos de notificación preferidos para revisiones sistemáticas y metanálisis (PRISMA, por su sigla en inglés). Las búsquedas se realizaron en las bases LILACS, PubMed, Scopus y SciELO en julio del 2017, sin limitaciones de tiempo. En todas las bases se utilizó la siguiente estrategia de búsqueda: (climate) AND (disease) AND (sensitive). Las búsquedas se realizaron en inglés, español y portugués.

Resultados. Se seleccionaron 106 publicaciones. Las enfermedades más estudiadas fueron el dengue, la malaria y las enfermedades cardiovasculares y respiratorias. Las variables climáticas más estudiadas fueron la temperatura y las precipitaciones. Los estudios mostraron una relación entre la incidencia de determinadas de enfermedades, principalmente de las enfermedades cardiovasculares y respiratorias, el dengue, la malaria y las enfermedades arbovirales, y las condiciones climáticas en diferentes regiones del mundo. Esa relación se analizó tanto con datos pasados de incidencia de enfermedades y variables climáticas como con una proyección de la incidencia futura de enfermedades, de acuerdo con las variaciones previstas del clima. Se encontró un mayor número de estudios realizados por autores oriundos de países desarrollados. Los lugares estudiados con mayor frecuencia fueron Australia, Brasil, China y Estados Unidos. Conclusiones. A pesar del aumento del número de artículos publicados sobre el tema, es preciso enfocarse en un mayor número de variables climáticas y ambientales, y ampliar los estudios a otras regiones del mundo.

Cambio climático; efectos del clima; dengue; malaria; enfermedades respiratorias; enfermedades cardiovasculares; Brasil. 